\title{
A SEPARATION THEOREM IN DIMENSION 3
}

\author{
F. ACQUISTAPACE, F. BROGLIA AND E. FORTUNA*
}

\section{Introduction}

Let $M$ be a compact non-singular real affine algebraic variety and let $A, B$ be open disjoint semialgebraic subsets of $M$. Define $Z={\overline{\bar{A}} \cap \bar{B}^{Z} \text { (where }}^{-Z}$ denotes the Zariski closure).

The sets $A, B$ are said generically separated if there exists a proper algebraic subset $X \subset M$ and a polynomial function $p \in \mathscr{P}(M)$ (or equivalently a regular function $p \in \mathscr{R}(M))$ such that $p(A-X)>0$ and $p(B-X)<0$.

The sets $A, B$ are said separated if there exists $p \in \mathscr{P}(M)$ such that $p(A-Z)>0$ and $p(B-Z)<0$.

Very general results on the problem of polynomial separation for semialgebraic sets are known, for instance Bröcker (cf. [Br 1], [Br 2]) solves the problem of the separation of constructible sets in a space of orderings. A detailed exposition of this subject can be found in $[\mathrm{AnBrRz}]$, where, in particular, general criterions for the separation of closed semialgebraic sets are given, by applying powerful tools of real algebra and quadratic forms theory.

We are interested in finding a finite number of geometric conditions equivalent to the separation of two open semialgebraic sets going towards an algorithmic solution of the problem. In this article we consider the case of a compact non-singular algebraic variety $M$ of dimension 3 .

The paper is structured as follows. Section 1 contains some general separation results for compact semialgebraic subsets of $\mathbf{R}^{n}$. Geometric obstructions to separation are found in Section 2, but the proof that this finite set of conditions is equivalent to the separation is postponed to Section 4 . In Section 3 we discuss the relations between separation and generic separation in dimension 3: if $A$ and $B$ can be separated outside $X$, then they can be separated outside a set $W$ which is

Received July $11,1995$.

* Members of G. N. S. A. G. A. of C. N. R.

The authors are partially supported by M. U. R. S. T. and Eurocontract ERBCHRXCT 940506. 
"the best possible", in the sense that any polynomial function generically separating $A$ from $B$ must vanish on $W$. Finally in Section 5 we give a criterion of separation working essentially when the Zariski boundaries of the sets $A$ and $B$ have only non-singular normal crossings components. So, up to desingularization, this criterion reduces the separation problem in dimension 3 to a separation problem on the Zariski boundaries of the sets, hence to a finite number of tests, It is a first step to prove the "decidability" of this problem.

\section{A separation tool}

Recall the following result which makes it possible to pass from local to global separation; it can be found in $[\mathrm{F}]$.

Proposition 1.1. Let $F, G$ be compact semialgebraic subsets of $\mathbf{R}^{n}$ such that $F \cap G=\{O\}$. Assume there exist a neighbourhood $U$ of $O$ and a polynomial function p such that

$$
p(F \cap U-\{O\})>0 \text { and } p(G \cap U-\{O\})<0 .
$$

Then $F$ and $G$ can be separated.

As a consequence we have:

Proposition 1.2. Let $F, G$ be compact semialgebraic subsets of $\mathbf{R}^{n}, \stackrel{\circ}{F} \cap \stackrel{\circ}{G}=$ $\emptyset$ and let $X$ be an algebraic subset of $\mathbf{R}^{n}$ such that $F \cap G \subseteq X$. Assume there exist a neighbourhood $U$ of $X$ and a polynomial function $p$ such that

$$
p(F \cap U-X)>0 \text { and } p(G \cap U-X)<0 .
$$

Then there exists a polynomial function $q$ such that

$$
q(F-X)>0 \text { and } q(G-X)<0 .
$$

Proof. Let $\pi: \mathbf{R}^{n} \rightarrow N$ be the topological contraction of $X$ to a point, say $O$. It is known (see [BoCRy]) that $N$ admits an affine algebraic structure such that $\pi$ becomes a regular function and $\left.\pi\right|_{\mathbf{R}^{n}-X}: \mathbf{R}^{n}-X \rightarrow N-\{O\}$ a biregular isomorphism. The sets $\pi(F)$ and $\pi(G)$ are compact semialgebraic sets and $\pi(F) \cap \pi(G)=$ $\{O\}$, since $F \cap G \subseteq X$. The function $p^{\circ}\left(\left.\pi\right|_{\mathbf{R}^{n}-X}\right)^{-1}: N-\{O\} \rightarrow \mathbf{R}$ is regular, so it can be written as $\frac{\varphi}{\phi}$, with $\varphi, \phi \in \mathscr{P}(N), \phi$ never vanishing on $N-\{O\}$. 
Then $\varphi \cdot \psi$ is a polynomial function on $N$ which separates $\pi(F)$ from $\pi(G)$ in the neighbourhood $\pi(U)$ of $O$.

$N$ is affine, say $N \subset \mathbf{R}^{m} ; \varphi \cdot \psi$ is the restriction of a polynomial function $q$ which verifies the hypothesis of Proposition 1.1. Hence there exists $f \in \mathscr{P}(N)$ such that

$$
f(\pi(F)-\{O\})>0 \text { and } f(\pi(G)-\{O\})<0 .
$$

Then $f \circ \pi$ is a regular function separating $F$ from $G$ outside $X$. If $f \circ \pi=\frac{q_{1}}{q_{2}}$, with $q_{1}, q_{2} \in \mathscr{P}\left(\mathbf{R}^{n}\right)$, then $q_{1} \cdot q_{2}$ is the polynomial function we looked for.

Proposition 1.3. Let $F, G$ be compact semialgebraic subsets of $\mathbf{R}^{n}, \stackrel{\circ}{F} \cap \stackrel{\circ}{G}=$ $\emptyset$ and let $X \subset \mathbf{R}^{n}$ be an algebraic set such that $F \cap G \subseteq X$. Denote by $X_{1}, \ldots, X_{r}$ the irreducible components of $X$ and assume that, for each $i \in\{1, \ldots, r\}$, there exist a neighbourhood $U_{\imath}$ of $X_{\imath}$ and a polynomial function $p_{\imath}$ such that

$$
p_{i}\left(F \cap U_{i}-X\right)>0 \text { and } p_{i}\left(G \cap U_{i}-X\right)<0 .
$$

Then there exists $q \in \mathscr{P}\left(\mathbf{R}^{n}\right)$ that $X$-separates $F$ from $G$, meaning by this that

$$
q(F-X)>0 \quad \text { and } \quad q(G-X)<0
$$

Proof. By Proposition 1.2, it is enough to prove that there exist a neighbourhood $U$ of $X$ and $p \in \mathscr{P}\left(\mathbf{R}^{n}\right)$ such that $p(F \cap U-X)>0$ and $p(G \cap U-X)$ $<0$.

This result will be achieved in some steps.

Define $X^{1}=\cup_{i \neq j}\left(X_{i} \cap X_{j}\right)$.

Step 1. Construction of a polynomial function $X$-separating $F$ from $G$ in a neighbourhood $W$ of $X-X^{1}$.

For each $i \in\{1, \ldots, r\}$, let $f_{\imath}$ be a positive equation of $X_{i}$ (i.e. $f_{\imath} \geq 0$ on $\mathbf{R}^{n}$, $\left.V\left(f_{\imath}\right)=X_{i}\right)$. Up to shrink it, we can assume that $U_{i}$ is a closed semialgebraic set. For each $i$, on $(F \cup G) \cap U_{i}$ the zero-set $V\left(p_{\imath}\right)$ is contained in $X$, which is the zero-set of $f_{1} \cdot \ldots \cdot f_{r}$. By Lojasiewicz inequality there exists an integer $n_{i}$ such that the rational function $\frac{\left(f_{1} \cdot \ldots \cdot f_{r}\right)^{n_{i}}}{p_{i}}$, extended to 0 on $V\left(p_{t}\right) \cap(F \cup G) \cap U_{t}$, is continuous on $(F \cup G) \cap U_{i}$. Take $m>n_{\imath}$, for each $i \in\{1, \ldots, r\}$. Then the function $\frac{\left(f_{1} \cdot \ldots \cdot f_{r}\right)^{m}}{p_{\imath}}$ is continuous and vanishes on $X \cap U_{i}$. We want to prove 
that the polynomial function

$$
P_{m}=f_{1}^{m} \cdot \ldots \cdot f_{r}^{m} \cdot\left(\sum_{i=1}^{r} \frac{p_{i}}{f_{i}^{m}}\right)
$$

$X$-separates $F$ from $G$ in a suitable neighbourhood $W$ of $X-X^{1}$.

In fact, take $x_{0} \in X_{i}-X^{1}$. Since $\frac{\left(f_{1} \cdot \ldots \cdot f_{r}\right)^{m}}{p_{i}}\left(x_{0}\right)=0$ and for all $j \neq i$ $f_{i}\left(x_{0}\right) \neq 0$, then $\lim _{x \rightarrow x_{0}} \frac{\left|p_{i}(x)\right|}{f_{i}^{m}(x)}=+\infty$. On the contrary $\sum_{j \neq i} \frac{p_{j}}{f_{j}^{m}}$ is bounded locally at $x_{0}$. So there exists a neighbourhood $U\left(x_{0}\right)$ of $x_{0}$ such that, on $U\left(x_{0}\right), P_{m}$ has the same sign as $p_{\imath}$. If we take $W_{\imath}=\cup_{x_{0} \in X_{i}-X^{1}} U\left(x_{0}\right)$, which is a neighbourhood of $X_{l}-X^{1}$, we have that $P_{m}$ has the same sign as $p_{i}$ on $W_{i}$; so $P_{m} X$-separates $F$ from $G$ in $W_{i}$. It is then enough to take $W=\cup_{i=1}^{r} W_{i}$.

Step 2. Proof of the statement in the case $\operatorname{dim} X^{1}=0$.

In this case $X^{1}$ is a finite set of points $\left\{Q_{1}, \ldots, Q_{r(1)}\right\}$ and, for each $j=1, \ldots$, $r(1)$, there exist a bounded neighbourhood $V_{j}$ of $Q_{j}$ and a polynomial function $q_{j} X$-separating $F \cap V_{j}$ from $G \cap V_{j}$; of course, we can suppose the neighbourhoods $V_{j}$ pairwise disjoint. Moreover, by Step 1 , we have a neighbourhood $W$ of $X$ $-\left\{Q_{1}, \ldots, Q_{r(1)}\right\}$ and $p \in \mathscr{P}\left(\mathbf{R}^{n}\right)$ that $X$-separates $F \cap W$ from $G \cap W$.

By suitable manipulations of $p$ and $q_{j}$, s, we will iteratively find a neighbourhood $W_{j}$ of $X-\left\{Q_{j+1}, \ldots, Q_{r(1)}\right\}$ and $p^{j} \in \mathscr{P}\left(\mathbf{R}^{n}\right) X$-separating $F \cap W_{j}$ from $G \cap W_{\text {, Then }} p^{r(1)}$ will $X$-separate $F$ from $G$ in a neighbourhood of $X$.

Take $j=1$; let $f$ be a positive equation of $X$ and $r_{1}$ a positive equation of $Q_{1}$ such that $\left\{r_{1} \leq 1\right\} \subseteq V_{1}$. Define $\overline{q_{1}}=\sup _{(F \cup G) \cap\left(\bar{W}-V_{1}\right)}\left|q_{1}\right|$. Up to shrink $W$ a little, we have that on $(F \cup G) \cap\left(\bar{W}-V_{1}\right)$

$$
V\left(\frac{p r_{1}}{\overline{q_{1}}}\right) \subseteq V(f)
$$

So by Lojasiewicz inequality there exists an integer $n$ such that, by taking a sufficiently small neighbourhood $W_{0}$ of $X-\left\{Q_{1}, \ldots, Q_{r(1)}\right\}$ one has

$$
f^{n} \leq \frac{r_{1}}{\overline{q_{1}}}|p| \quad \text { on } \quad(F \cup G) \cap\left(\bar{W}_{0}-V_{1}\right)
$$

and therefore, for any $m \in \mathbf{N}$,

$$
\left|q_{1}\right| f^{n} \leq r_{1}|p| \leq r_{1}^{m}|p| \quad \text { on } \quad(F \cup G) \cap\left(\bar{W}_{0}-V_{1}\right) .
$$

Then, for any positive integer $m$, the polynomial function $r_{1}^{m} p+f^{n} q_{1}$ has the same 
sign as $p$ on $(F \cup G) \cap\left(\bar{W}_{0}-V_{1}\right)$.

Now consider the set $(F \cup G) \cap\left(\bar{V}_{1}-W_{0}\right)$, on which $V\left(\frac{f^{n} q_{1}}{p}\right) \subseteq V\left(r_{1}\right)$, where $\bar{p}=\sup _{(F \cup G) \cap\left(\overline{V_{1}}-W_{0}\right)}|p|$. So there exists $m \in \mathbf{N}$ (depending on $n$ ) such that, by taking a sufficiently small neighbourhood $V_{1}^{\prime}$ of $Q_{1}$, on $(F \cup G) \cap\left(\overline{V_{1}^{\prime}}-W_{0}\right)$ we have $r_{1}^{m} \leq \frac{f^{n}}{p}\left|q_{1}\right|$, and therefore $r_{1}^{m}|p| \leq f^{n}\left|q_{1}\right|$. Hence $p^{1}=r_{1}^{m} p+f^{n} q_{1}$ has the same sign as $q_{1}$ on $\overline{V_{1}^{\prime}}-W_{0}$. Since $p^{1}$ clearly $X$-separates $F$ and $G$ on $V_{1} \cap W$, then it $X$-separates $F$ and $G$ in $\left(\bar{W}_{0}-V_{1}\right) \cup\left(\overline{V_{1}^{\prime}}-W_{0}\right) \cup\left(V_{1} \cap W\right)$, which is a neighbourhood of $X-\left\{Q_{2}, \ldots, Q_{r}\right\}$.

By the same argument we can find the polynomials $p^{2}, \ldots, p^{r(1)}$ as planned above.

Step 3. Proof of the Proposition in the general case.

Consider the decreasing sequence of algebraic sets

$$
X \supset X^{1} \supset X^{2} \supset \cdots \supset X^{s}
$$

where $X=X_{1} \cup X_{2} \cup \cdots \cup X_{r}, X^{1}=\cup_{i \neq j}\left(X_{i} \cap X_{j}\right)$ and recursively if $X_{1}^{\beta} \cup$ $\cdots \cup X_{r(\beta)}^{\beta}$ is the decomposition into irreducible components of $X^{\beta}, X^{\beta+1}=\cup_{i \neq j}$ $\left(X_{\imath}^{\beta} \cap X_{j}^{\beta}\right)$.

Clearly $\operatorname{dim} X^{\beta}<\operatorname{dim} X^{\alpha}$ if $\beta>\alpha$, so we can assume $X^{s} \neq \varnothing$ and $X^{s+1}=$ $\emptyset$. We will recursively find neighbourhoods $W^{\beta}$ of $X-X^{\beta+1}$ and polynomial functions $p_{\beta}$ such that $p_{\beta}\left(F \cap W^{\beta}-X\right)>0$ and $p_{\beta}\left(F \cap W^{\beta}-X\right)<0$. Clearly $p_{s}$ will $X$-separate $F$ from $G$ in a neighbourhood of $X$ and the thesis will be a consequence of Proposition 1.2.

By Step 1 , we know that $F$ and $G$ are $X$-separated by $p \in \mathscr{P}\left(\mathbf{R}^{n}\right)$ in a neighbourhood $W$ of $X-X^{1}$.

From the hypothesis, it follows that for each $j \in\{1, \ldots, r(1)\}$ there exist a neighbourhood $V_{j}$ of $X_{j}^{1}$ and $q_{j} \in \mathscr{P}\left(\mathbf{R}^{n}\right)$ such that $q_{j}\left(F \cap V_{j}-X\right)>0$ and $q_{j}\left(G \cap V_{j}-X\right)<0$. Let $f$ be a positive equation of $X$ and $r_{1}$ a positive equation of $X_{1}^{1}$ such that $\left\{r_{1} \leq 1\right\} \subseteq V_{1}$.

Define $\overline{q_{1}}=\sup _{(F \cup G) \cap\left(\bar{W}-V_{1}\right)}\left|q_{1}\right|$. By the same argument used in Step 2, there exists $n \in \mathbf{N}$ such that, for any $m \in \mathbf{N}, f^{n} q_{1}+r_{1}^{m} p$ has the same sign as $p$ on $(F \cup G) \cap\left(\bar{W}_{0}-V_{1}\right)$, where $W_{0}$ is a sufficiently small neighbourhood of $X-$ $X^{1}$

Consider now a neighbourhood $V_{1}^{\prime}$ of $X_{1}^{1}-X^{2}, V_{1}^{\prime} \subset V_{1}$ and such that $V_{1}^{\prime} \cap$ $X_{j}^{1}=\varnothing$ for each $j \neq 1$. On $(F \cup G) \cap\left(\overline{V_{1}^{\prime}}-W_{0}\right)$ we have that $V\left(\frac{f^{n} q_{1}}{\bar{p}}\right) \subseteq$ 
$V\left(r_{1}\right)$, where $\bar{p}=\sup _{(F \cup G) \cap\left(\overline{V_{1}^{\prime}}-W_{0}\right)}|p|$.

So there exists $m$ (depending on $n$ ) such that, possibly after shrinking $V_{1}^{\prime}$, on $(F \cup G) \cap\left(\overline{V_{1}^{\prime}}-W_{0}\right)$ we have $r_{1}^{m} \leq \frac{f^{n}\left|q_{1}\right|}{p}$, and therefore $r_{1}^{m}|p| \leq f^{n}\left|q_{1}\right|$.

So $p^{1}=r_{1}^{m} p+f^{n} q_{1} X$-separates $F$ from $G$ in $\overline{V_{1}^{\prime}}-W_{0}$. Since $p$ and $q_{1}$ have the same sign on $(F \cup G) \cap\left(W_{0}-V_{1}\right)$, we get that $p^{1} X$-separates $F$ from $G$ in a neighbourhood $W_{1}$ of $\left(X-X^{1}\right) \cup\left(X_{1}^{1}-X^{2}\right)$.

We can repeat the above argument replacing $W$ by $W_{1}, X_{1}^{1}$ by $X_{2}^{1}, V_{1}$ by $V_{2}$ and $q_{1}$ by $q_{2}$. So we find a neighbourhood $W_{2}$ of $\left(X-X^{1}\right) \cup\left(X_{1}^{1} \cup X_{2}^{1}-X^{2}\right)$ and a polynomial function $p^{2}$ which $X$-separates $F \cap W_{2}$ from $G \cap W_{2}$.

Repeating this procedure, eventually we find a neighbourhood $W^{1}$ of $X-X^{2}$ and $p_{1} \in \mathscr{P}\left(\mathbf{R}^{n}\right)$ such that $p_{1}\left(F \cap W^{1}-X\right)>0$ and $p_{1}\left(G \cap W^{1}-X\right)<0$.

By iterating this argument, we construct successively the polynomials $p_{2}, \ldots$, $p_{s}$ as described above.

\section{Obstructions}

Let $M$ be a compact, non-singular, real affine algebraic variety, $\operatorname{dim} M=3$, and let $A, B$ be open disjoint semialgebraic subsets of $M$.

We will denote by $Y$ the algebraic set $\overline{\partial A}^{Z} \cup \overline{\partial B}^{Z}$, by $Y_{1}, \ldots, Y_{k}$ the irreducible components of $Y$ of dimension 2 and by $Z$ the set $\overline{\bar{A} \cap \bar{B}^{Z}}$.

\section{Definition 2.1.}

a) We say that $p \in \mathscr{R}(M)$ changes its sign at $x \in M$ if, for every neighbourhood $V$ of $x$, there exist $y_{1}, y_{2} \in V$ such that $p\left(y_{1}\right) p\left(y_{2}\right)<0$.

b) Let $X \subset M$ be a 2 -dimensional algebraic set and let $p \in \mathscr{R}(M)$. We say that $p$ changes its sign across $X$ if it changes its sign at any point $x \in X$ such that $\operatorname{dim} X_{x}=2$.

Definition 2.2. We say that an irreducible component $Y_{\imath}$ of $Y, i \in\{1, \ldots, k\}$, is odd (resp. even) if there exists an open set $\Omega \subseteq M$ such that $\operatorname{dim}\left(Y_{\imath} \cap \Omega\right)=2$, $A \cap \Omega$ and $B \cap \Omega$ can be generically separated and every $p \in \mathscr{R}(M)$ generically separating them changes (resp. does not change) its sign across $Y_{i}$. An irreducible component $Y_{i}$ of $Y$ will be called a 2 -obstruction if it is both odd and even. 


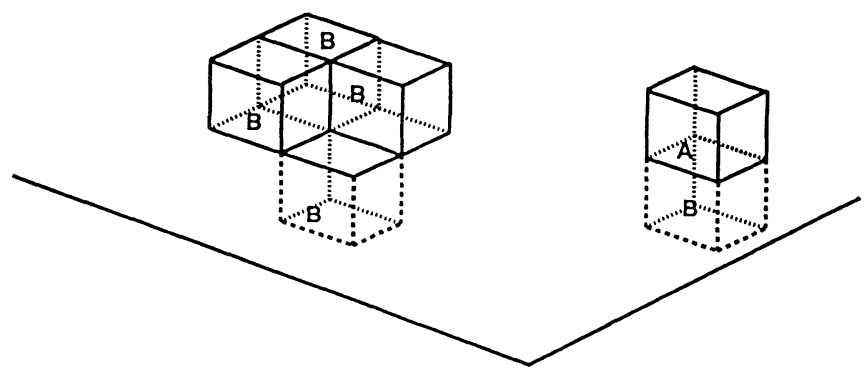

Fig. 1. An example of a 2-obstruction

Remark 2.3. In Definition 2.2 we can suppose that $\mathscr{g}\left(Y_{i}\right) \mathscr{R}(\Omega)$ is a principal ideal, since this is true on a suitable Zariski open set $M-X$. Let $g$ be a generator. Then if $Y_{\imath}$ is odd (resp. even), any regular function $p$ generically separating $A \cap \Omega$ from $B \cap \Omega$ can be written as $p=g^{m} q$, with $q \notin g\left(Y_{i}\right) \mathscr{R}(\Omega)$, and $m$ odd (resp. even, possibly zero). It is also clear that the parity of $m$ does not depend on the choice of the Zariski open set and of the generator.

Notation 2.4. Let $A$ and $B$ be open semialgebraic sets and $g$ be a regular function on $M$. Denote by $A_{g}$ and $B_{g}$ the sets

$$
\begin{aligned}
& A_{g}=(A \cap\{g>0\}) \cup(B \cap\{g<0\}) \\
& B_{g}=(A \cap\{g<0\}) \cup(B \cap\{g>0\}) .
\end{aligned}
$$

LEMma 2.5. Let $g$ be a regular function on $M$ such that

- for any $\alpha \in\{1, \ldots, r\}, g \in g\left(Y_{\alpha}\right)$ and $g$ changes its sign across $Y_{\alpha}$

- for any $\alpha \in\{r+1, \ldots, k\}, g \notin g\left(Y_{\alpha}\right)$.

Then

- for any $\alpha \in\{1, \ldots, r\}, Y_{\alpha}$ is odd (resp. even) with respect to $A, B \Leftrightarrow Y_{\alpha}$ is even (resp. odd) with respect to $A_{g}, B_{g}$

- for any $\alpha \in\{r+1, \ldots, k\}, Y_{\alpha}$ is odd (resp. even) with respect to $A, B \Leftrightarrow Y_{\alpha}$ is odd (resp. even) with respect to $A_{g}, B_{g}$.

Proof. If $p \in R(M)$ generically separates $A \cap \Omega$ from $B \cap \Omega$, that is

$$
p(A \cap \Omega-X)>0 \text { and } p(B \cap \Omega-X)<0,
$$

then

$$
\operatorname{pg}\left(A_{g} \cap \Omega-X\right)>0 \quad \text { and } \operatorname{pg}\left(B_{g} \cap \Omega-X\right)<0
$$


i.e. $p g$ generically separates $A_{g} \cap \Omega$ from $B_{g} \cap \Omega$.

Moreover, for any $p^{\prime}$ generically separating $A_{g} \cap \Omega$ from $B_{g} \cap \Omega$, we have

$$
p^{\prime}\left(A_{g} \cap \Omega-X\right)>0 \text { and } p^{\prime}\left(B_{g} \cap \Omega-X\right)<0 \text {, }
$$

then

$$
p^{\prime} g(A \cap \Omega-(X \cup V(g)))>0 \text { and } p^{\prime} g(B \cap \Omega-(X \cup V(g)))<0 .
$$

Hence $p^{\prime} g$ generically separates $A \cap \Omega$ from $B \cap \Omega$

Assume, for instance, $Y_{\imath}$ is odd with respect to $A, B$. Then, for any $p^{\prime}$ generically separating $A_{g} \cap \Omega$ from $B_{g} \cap \Omega$, $p^{\prime} g$ changes its sign across $Y_{i}$.

Since by hypothesis $g$ changes its sign across $Y_{1}, \ldots, Y_{r}$ and does not change it across $Y_{r+1}, \ldots, Y_{k}$, then:

if $i \in\{1, \ldots, r\}, p^{\prime}$ does not change its sign across $Y_{\imath}$, i.e. $Y_{\imath}$ is even with respect to $A_{g}, B_{g}$.

if $i \in\{r+1, \ldots, k\}, p^{\prime}$ changes its sign across $Y_{i}$, i.e. $Y_{\imath}$ is even with respect to $A_{g}, B_{g}$.

Arguing in the same way, one easily complete the proof.

Notation 2.6. We will denote by $Y^{\mathrm{c}}$ the union of the odd components of $Y$ (with respect to $A, B$ ).

Since any regular function separating $A$ from $B$ must vanish on $Y^{\mathrm{c}}$, if $Y^{\mathrm{c}} \cap$ $(A \cup B$ ) is not contained in $Z$, evidently $A$ and $B$ cannot be separated in the sense of the classical definition.

Now we can state a result which will be proved in Section 4 .

THEOREM 2.7. Let $M$ be a compact, non-singular, real affine algebraic variety, $\operatorname{dim} M=3$, and let $A, B$ be open disjoint semialgebraic subsets of $M$. Define $Y=$ $\overline{\partial A}^{Z} \cup \overline{\partial B}^{Z}$ and $Z={\overline{\bar{A} \cap \bar{B}^{2}}}^{Z}$.

Then $A$ and $B$ can be separated if and only if the following conditions hold:

1) No 2-dimensional irreducible component $Y_{i}$ of $Y, i \in\{1, \ldots, k\}$, is a 2-obstruction.

2) For every $T_{\jmath}, j \in\{1, \ldots, s\}$, irreducible component of Sing $Y$, there exists an open semialgebraic neighbourhood $U_{j}$ of $T$, such that $A \cap U_{j}$ and $B \cap U$, can be separated.

3) $Y^{c} \cap(A \cup B) \subseteq Z$. 
Examples 2.8. In the example in Fig. 2 condition 2) fails; in the example in Fig. 3 (taken from $[\mathrm{Br} 1]$ ) neither condition 1) nor condition 2) are verified.

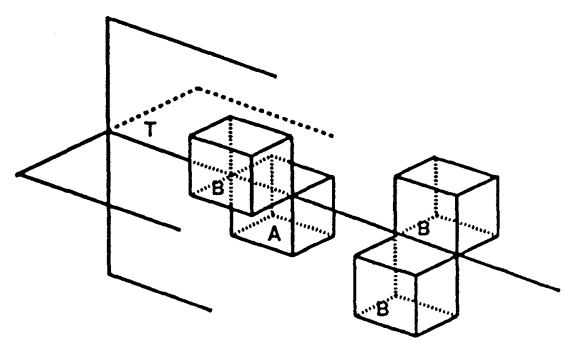

Fig. 2

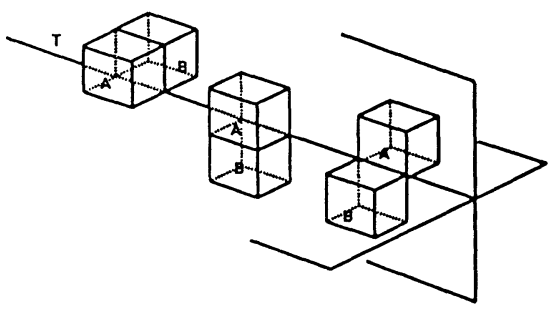

Fig. 3

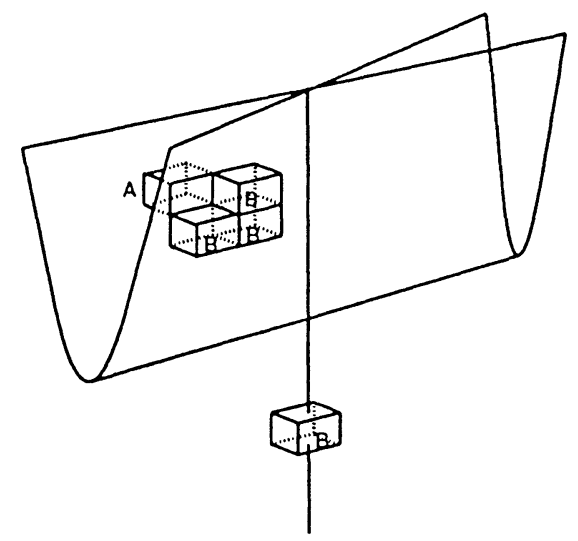

Fig. 4

In the example in Fig. 4 condition 3) fails, because $Y^{c}$ is the whole Whitney umbrella while $Z$ is a 1 -dimensional algebraic subset of $Y^{c}$ not containing the stick of the umbrella.

Remark 2.9. If there are no 2-obstructions, then $\operatorname{dim} Y^{c} \cap(A \cup B) \leq 1$, therefore $Y^{c}$ can intersect $A \cup B$ only with its "tails". For instance, if $Y$ is a union of non-singular irreducible components and condition 1) holds, then $Y^{c} \cap$ $(A \cup B)=\emptyset$.

\section{Separation and generic separation in dimension 3}

First, let us recall two results we shall use later on. 
Theorem 3.1 (Bröcker-Lojasiewicz, [BoCRy] 7.7.10). Let $S$ be a closed semialgebraic subset of a real algebraic variety $V$ and let $f, g$ be regular functions on $V$. Then there exists a non-negative regular function $\varepsilon$ such that:

$-(f+\varepsilon g)(x)$ has the same sign as $f(x)$, for any $x \in S$

$-V(\varepsilon) \subseteq \overline{V(f) \cap S}$

THEOREM 3.2 (Ruiz, $[\mathrm{Rz}]$ ). Let $U$ be a 1-dimensional open semialgebraic subset of a real algebraic variety $V$. Then there exists $h \in \mathscr{P}(V)$ such that:

$$
U=\{x \in V \mid h(x)>0\} \text { and } \bar{U}=\{x \in V \mid h(x) \geq 0\} .
$$

It is well known that generic separation and separation are equivalent in dimension 2 (as one can prove using Theorems 3.1 and 3.2): Fig. 4 shows this is not true in dimension 3 .

As we remarked before, any regular function generically separating $A$ from $B$ must vanish on $Y^{c} \cup Z$. In this section we will prove that this "lower bound" for $V(f) \cap(\bar{A} \cup \bar{B})$ can always be attained:

THEOREM 3.3. If $A$ and $B$ can be generically separated, then there exists $f \in$ $\mathscr{R}(M)$ such that

$$
\begin{gathered}
f\left(A-\left(Z \cup Y^{c}\right)\right)>0, f\left(B-\left(Z \cup Y^{c}\right)\right)<0 \text { and } \\
V(f) \cap(\bar{A} \cup \bar{B})=\left(Z \cup Y^{c}\right) \cap(\bar{A} \cup \bar{B}) .
\end{gathered}
$$

Proof. By hypothesis, there exist an algebraic subset $X$ of $M, \operatorname{dim} X \leq 2$, and $p \in \mathscr{R}(M)$ such that $p(A-X)>0$ and $p(B-X)<0$. Clearly we can assume $X=\overline{X \cap(A \cup B)}^{Z}$; in particular no irreducible 2-dimensional component of $X$ lies in $Y^{c}$.

Let $X^{\prime}$ denote the union of the irreducible components of $X$ of dimension 2 . Since $p$ does not change its sign across any component of $X^{\prime}, p \in \mathscr{g}\left(X^{\prime}\right)^{2}$ (for a proof see $[\mathrm{AcBg}])$. So we can write $p=g^{k} p^{\prime}$, where $g$ is a generator of $\mathscr{g}\left(X^{\prime}\right)^{2}, p^{\prime}$ $\in \mathscr{R}(M)$ and $p^{\prime} \notin g\left(X^{\prime}\right)^{2}$. The function $p^{\prime}$ does not change its sign across $X^{\prime}$, so $p^{\prime} \notin \mathscr{g}\left(X^{\prime}\right)$, i.e. $\left.p^{\prime}\right|_{X^{\prime}} \not \equiv 0$. Then, up to replace $p$ by $p^{\prime}$, we can suppose $\operatorname{dim} X \leq 1$.

Consider now all the 2-dimensional irreducible components of $Y$, say $Y_{1}, \ldots$, $Y_{l}$, which do not lie in $Y^{c}$ and on which $p$ identically vanishes (after the first reduction we have made, such components can intersect $A \cup B$ only in dimension 1). For any $\alpha \in\{1, \ldots, l\}$, since $A$ and $B$ can be generically separated and $Y_{\alpha}$ is not odd, there exists $q_{\alpha} \in \mathscr{R}(M)$ generically separating $A$ from $B$ which does not change its sign across $Y_{\alpha}$. We can suppose that $q_{\alpha}$ does not vanish on $Y_{\alpha}$; in fact 
if $q_{\alpha \mid Y_{\alpha}} \equiv 0$, then $q_{\alpha} \in \mathscr{J}\left(Y_{\alpha}\right)^{2}$, which enables us to use the same factorization argument as above.

Then the regular function $\sum_{\alpha=1}^{l} q_{\alpha}$ separates $A-\cap_{\alpha=1}^{l} V\left(q_{\alpha}\right)$ from $B-\cap_{\alpha=1}^{l} V\left(q_{\alpha}\right)$ and does not vanish identically on $Y_{1} \cup \cdots \cup Y_{l}$. Hence $p+$ $\sum_{\alpha=1}^{l} q_{\alpha}$ separates $A-X$ from $B-X$ and does not vanish identically on $Y_{1} \cup \cdots \cup$ $Y_{l}$. Therefore, up to replace $p$ by $p+\sum_{\alpha=1}^{l} q_{\alpha}$, we can assume that $V(p) \cap$ $(\bar{A} \cup \bar{B})-\left(Z \cup Y^{c}\right) \leq 1$.

Consider now the semialgebraic set

$$
L=V(p) \cap \bar{A}-\left(Z \cup Y^{c}\right) .
$$

We know that $\operatorname{dim} L \leq 1$, so assume first that $\operatorname{dim} L=1$. Then there exists a finite set $\Gamma \subset L$ such that $L-\Gamma$ is open in $\bar{L}^{Z}$. By Theorem 3.2, we can find $h \in$ $\mathscr{P}(M)$ such that

$$
L-\Gamma=\left\{x \in \bar{L}^{Z} \mid h(x)>0\right\} \text { and } \overline{L-\Gamma}=\left\{x \in \bar{L}^{Z} \mid h(x) \geq 0\right\} .
$$

In particular, $h$ is strictly negative on $V(p) \cap \bar{B}-\left(Z \cup Y^{c}\right)$, because $\overline{L-\Gamma} \subset$ $\bar{A}$ and $\bar{A} \cap \bar{B} \subseteq Z$.

Consider the closed semialgebraic set

$$
S=(\bar{A} \cap\{h \leq 0\}) \cup(\bar{B} \cap\{h \geq 0\})
$$

and apply Theorem 3.1 to $p, h$ and $S$. We get $\varepsilon \in \mathscr{R}(M), \varepsilon \geq 0$, such that $\varphi=p$ $+\varepsilon h$ has the same sign as $p$ on $S$ and $V(\varepsilon) \subseteq \overline{V(p) \cap S}^{Z}$. In particular $\varphi(\bar{A}) \geq 0$ and $\varphi(\bar{B}) \leq 0$. Moreover,

$$
V(\varphi) \cap(\bar{A} \cup \bar{B})=(V(\varphi) \cap S) \cup(V(\varphi) \cap(\bar{A} \cup \bar{B})-S) ;
$$

but

$$
\begin{gathered}
V(\varphi) \cap S=V(p) \cap S=(V(p) \cap \bar{A} \cup\{h \leq 0\}) \cup(V(p) \cap \bar{B} \cap\{h \geq 0\}) \\
\subseteq \Gamma \cup Z \cup Y^{c}
\end{gathered}
$$

and

$$
V(\varphi) \cap(\bar{A} \cup \bar{B})-S \subseteq V(\varepsilon) \subseteq \overline{V(p) \cap S}^{Z} \subseteq \Gamma \cup Z \cup Y^{c}
$$

So

$$
V(\varphi) \cap(\bar{A} \cup \bar{B}) \subseteq \Gamma \cup Z \cup Y^{c}
$$

In order to remove the 0 -dimensional set $\Gamma$, it is enough to apply two more times Theorem 3.1: the first time to the functions $\varphi$ and 1 with respect to $\bar{B}$ to obtain a function $\psi$ which does not vanish any more on the points of $\Gamma \cap(\bar{A}-\bar{B})$; the 
second time to $\psi$ and -1 with respect to $\bar{A}$ to obtain a function $f$ such that

$$
V(f) \cap(\bar{A} \cup \bar{B}) \subseteq Z \cup Y^{c} .
$$

The last argument can be used also when $\operatorname{dim} L=0$.

Remark 3.4. The irreducible components of $Y$ of dimension $\leq 1$ have no influence on the possibility of separating $A$ from $B$. To see this, denote their union by $H$ and consider the sets $A^{\prime}=\widehat{A \cup H}$ and $B^{\prime}=\widehat{\circ \cup H}$. We easily see that $Z^{\prime}=Z, Y=Y^{\prime} \cup H$ and all the irreducible components of $Y^{\prime}$ have dimension 2 . Remark that $A$ and $B$ can be separated if and only if $A^{\prime}$ and $B^{\prime}$ can be separated. In fact one implication is obvious since $A \subseteq A^{\prime}$ and $B \subseteq B^{\prime}$; conversely if $A$ and $B$ are separated by $p$, then $p$ separates $A^{\prime}-H$ from $B^{\prime}-H$, so by Theorem 3.3 $A^{\prime}$ and $B^{\prime}$ can be separated outside $Z \cup Y^{c}$. This is the reason why in Theorem 2.7 , in order to obtain the separation of $A$ from $B$, it is enough to impose some conditions only on Sing $Y$ and the 2-dimensional components of $Y$, without assuming anything on the lower dimensional ones.

Corollary 3.5. If $A$ and $B$ can be generically separated and $Y^{c} \cap(A \cup B) \subseteq$ $Z$, then $A$ and $B$ can be separated. Moreover there exists $f$ separating $A$ from $B$ and such that $V(f) \cap(\bar{A} \cup \bar{B})=\left(Z \cup Y^{c}\right) \cap(\bar{A} \cup \bar{B})$.

Proof. It follows immediately from Theorem 3.3.

Theorem 3.3 assures that $A$ and $B$ can be generically separated if and only if the sets $\hat{A}=A-Y^{c}$ and $\hat{B}=B-Y^{c}$ can be separated. If we consider the sets $\hat{Y}$ and $\hat{Z}$ defined in an evident way with respect to $\hat{A}$ and $\hat{B}$, it is easy to see that $\hat{Y}=Y$ and $\hat{Z}=Z$. If we use Theorems 2.7 and 3.3 as a consequence we get:

Corollary 3.6. $A$ and $B$ can be generically separated if and only if the following conditions hold:

1) No 2-dimensional irreducible component $Y_{\imath}$ of $Y, i \in\{1, \ldots, k\}$, is a 2-obstruction.

2) For every $T, j \in\{1, \ldots, s\}$, irreducible component of $\operatorname{Sing} Y$, there exists an open semialgebraic neighbourhood $U$, of $T$, such that $A \cap U$, and $B \cap U_{j}$ can be generically separated. 


\section{Proof of Theorem 2.7}

If $A$ and $B$ can be separated, then obviously conditions 1), 2), 3) hold.

Conversely, assume that conditions 1), 2), 3) hold; the proof that $A$ and $B$ can be separated will be achieved in some steps.

Step 1. We can assume that $Y$ has only non-singular, normal crossings irreducible components.

Let $\pi: \tilde{M} \rightarrow M$ be a desingularization of $Y \subset M$. This means that, if we denote by $Y_{1}^{\prime}, \ldots, Y_{l}^{\prime}$ the strict transforms of all the irreducible components $Y_{1}, \ldots$, $Y_{l}$ of $Y$, we have that:

a) $Y_{1}^{\prime}, \ldots, Y_{l}^{\prime}$ are non-singular and pairwise disjoint,

b) $E=\pi^{-1}$ (Sing $Y$ ) has non-singular irreducible components and $E \cup Y_{1}^{\prime} \cup$ $\ldots \cup Y_{l}^{\prime}$ has only normal crossings,

c) $\pi$ is surjective and induces a biregular isomorphism between $\tilde{M}-E$ and $M-\operatorname{Sing} Y$.

Define $\tilde{A}=\pi^{-1}(A), \tilde{B}=\pi^{-1}(B)$ and $\tilde{Y}=\overline{\partial \tilde{A}}^{Z} \cup \overline{\partial \tilde{B}}^{Z}$. It is clear that $\tilde{Y} \subseteq$ $\pi^{-1}(Y)=E \cup Y_{1}^{\prime} \cup \ldots \cup Y_{l}^{\prime}$.

Let us see that $\tilde{A}$ and $\tilde{B}$ verify conditions 1$), 2), 3$ ).

In fact, the algebraic set $\tilde{Y}$ is contained in $E \cup Y^{\prime}$, where $Y^{\prime}$ is the strict transform of $Y$. So an irreducible component $X$ of $\tilde{Y}$ is either the strict transform of a component $Y_{1}$ of $Y$, or a component of the exceptional divisor.

In the first case, if $X$ has dimension 2, it cannot be a 2-obstruction for the separation of $\tilde{A}$ and $\tilde{B}$ since $Y_{\imath}$ is not a 2 -obstruction and $\pi$ is a biregular isomorphism outside $E$.

In the second one, $\pi(X) \subseteq \operatorname{Sing} Y$ has dimension 1 or 0 . So, by condition 2), there exists a polynomial function $p$ separating $A$ and $B$ in a neighbourhood of $\pi(X)$. Hence $p^{\circ} \pi$ separates $\tilde{A}$ and $\tilde{B}$ in a neighbourhood of $X$.

For the same reason no irreducible component of $\operatorname{Sing} \tilde{Y}$ can be an obstruc. tion, because it lies in at least one component of $E$. So $\tilde{A}$ and $\tilde{B}$ verify 1 ) and 2).

Moreover, since $\tilde{Y}$ has non-singular irreducible components, 3 ) is automatically verified (see Remark 2.9).

Now suppose $\tilde{A}$ and $\tilde{B}$ can be separated: then, by composition with $\pi^{-1}$ (where defined), we get that $A$ and $B$ are generically separated, so applying Corollary 3.5 they can be separated.

Let $X^{\prime}$ be an algebraic subset of $M$ such that $\left[Y^{c} \cup X^{\prime}\right]=0$ in $H_{2}\left(M, \mathbf{Z}_{2}\right)$. Being $Y^{c}$ a union of non-singular components, we can assume that $X^{\prime}$ is 
transversal to each irreducible component of $Y^{c}$ and of Sing $Y$ (see for instance [BoCRy], chap. 12).

Since (Sing $Y)-Y^{c} \cap Y^{c}$ is a discrete set of points, we can further choose $X^{\prime}$ not passing through such points. So, if we denote $\Gamma=Y^{c} \cap X^{\prime}$, we can assume that $\operatorname{dim} \Gamma \leq 1, \operatorname{dim}(\Gamma \cap \operatorname{Sing} Y) \leq 0$ and $\Gamma \cap \overline{(\operatorname{Sing} Y)-Y^{c^{2}}}=\emptyset$.

Similarily there exists an algebraic subset $X^{\prime \prime}$ of $M$ such that $\left[Y^{c} \cup X^{\prime \prime}\right]=$ 0 , transversal to each irreducible component of $Y^{c}$ and of $\operatorname{Sing} Y$, and "avoiding" the points of $\Gamma \cap \operatorname{Sing} Y$. More precisely we can assume $\operatorname{dim}\left(\Gamma \cap X^{\prime \prime}\right) \leq 0$ and $\Gamma \cap X^{\prime \prime} \cap \operatorname{Sing} Y=\emptyset$. So the set $\Gamma \cap X^{\prime \prime}$ consists of a finite number of points $Q_{1}, \ldots, Q_{s}$ lying in $Y^{\mathrm{c}}$ and each of them is a non-singular point for $Y$. We can suppose that each $Q$, is non-singular for $X^{\prime \prime}$ too.

Now let $g^{\prime \prime}$ be a generator of the ideal $\mathscr{g}\left(Y^{c} \cup X^{\prime \prime}\right)$ which exists since $\left[Y^{c} \cup\right.$ $\left.X^{\prime \prime}\right]=0$.

Consider the sets $A_{g^{\prime \prime}}$ and $B_{g^{\prime \prime}}$, which for simplicity we will denote respectively $A^{\prime \prime}$ and $B^{\prime \prime}$; define $Y^{\prime \prime}={\overline{\partial A^{\prime \prime}}}^{Z} \cup{\overline{\partial B^{\prime \prime}}}^{Z}$ and $Z^{\prime \prime}=\overline{\overline{A^{\prime \prime}} \cap \overline{B^{\prime \prime}}}$. It is easy to check that $Y^{\prime \prime} \subseteq Y \cup X^{\prime \prime}$. Moreover we claim that

$$
Z^{\prime \prime} \cap\left(A^{\prime \prime} \cup B^{\prime \prime}\right)=Z \cap\left(A^{\prime \prime} \cup B^{\prime \prime}\right) \text {. }
$$

In fact, since $\overline{A^{\prime \prime}} \cap \overline{B^{\prime \prime}} \subseteq(\bar{A} \cap \bar{B}) \cup V\left(g^{\prime \prime}\right)$, we get $Z^{\prime \prime} \subseteq Z \cup V\left(g^{\prime \prime}\right)$; in particu$\operatorname{lar} Z^{\prime \prime} \cap\left(A^{\prime \prime} \cup B^{\prime \prime}\right) \subseteq Z \cap\left(A^{\prime \prime} \cup B^{\prime \prime}\right)$.

Conversely, let $x \in Z \cap\left(A^{\prime \prime} \cup B^{\prime \prime}\right)$ and assume $H$ is an irreducible component of $Z$ passing through $x$. $H$ contains an open subset $U$ of $\bar{A} \cap \bar{B}$ of maximal dimension such that $H=\bar{U}^{Z}$. Since $g(x) \neq 0, g_{H} \not \equiv 0$ and also $g_{\mid \bar{U}} \not \equiv 0$; so $U \subseteq$ $\overline{A^{\prime \prime}} \cap \overline{B^{\prime \prime}}$ and therefore $H \subseteq Z^{\prime \prime}$. Then $x \in Z^{\prime \prime} \cap\left(A^{\prime \prime} \cup B^{\prime \prime}\right)$.

Assume $Y^{c}=Y_{1} \cup \ldots \cup Y_{r}$. By Lemma 2.5, the components $Y_{1}, \ldots, Y_{r}$ are even w.r.t. $A^{\prime \prime}, B^{\prime \prime}$, while $Y_{r+1}, \ldots, Y_{k}$ are not odd w.r.t. $A^{\prime \prime}, B^{\prime \prime}$, because they were not odd w.r.t. $A, B$. This means that no 2-dimensional irreducible component of $Y$ is odd w.r.t. $A^{\prime \prime}, B^{\prime \prime}$ and therefore that $\left(\overline{A^{\prime \prime}} \cap \overline{B^{\prime \prime}}\right)-X^{\prime \prime} \subseteq \operatorname{Sing} Y$; in other words $Z^{\prime \prime} \subseteq(\operatorname{Sing} Y) \cup X^{\prime \prime}$.

Step 2. $A^{\prime \prime}$ and $B^{\prime \prime}$ can be separated in a neighbourhood of $X^{\prime \prime} \cap \Gamma$.

For each $j \in\{1, \ldots, s\}, Q, \notin \operatorname{Sing} Y$, so there exists a neighbourhood $V_{j}$ of $Q_{j}$ such that $Y \cap V$, is contained in exactly one irreducible component of $Y$ (more precisely, of $Y^{c}$ ). We can assume the $V$,'s pairwise disjoint. Let $V=V_{1} \cup \cdots \cup$ $V_{s}$.

Since $\overline{A^{\prime \prime}} \cap \overline{B^{\prime \prime}} \subseteq(\operatorname{Sing} Y) \cup X^{\prime \prime}$, we have that $\overline{A^{\prime \prime}} \cap \overline{B^{\prime \prime}} \cap V \subseteq X^{\prime \prime}$. If the $V_{j}$ 's are small enough, also $X^{\prime \prime} \cap V$ consists of non-singular points for $X^{\prime \prime}$. Let $q$ 
be a regular function in $\mathscr{g}\left(X^{\prime \prime}\right)$ such that $V(q) \cap V=X^{\prime \prime} \cap V$ and $q$ changes its sign at any point of $X^{\prime \prime} \cap V$.

For each $j \in\{1, \ldots, s\}, A^{\prime \prime} \cap V_{j}$ and $B^{\prime \prime} \cap V$, are separated by $q$ or $-q$. Then we can suppose that $q$ separates $A^{\prime \prime} \cap V$ from $B^{\prime \prime} \cap V$ (up to multiplying $q$ by the equation of a sphere centered in $Q_{i}$ and containing $V_{v}$, for each $i$ such that $A^{\prime \prime} \cap V_{i}$ and $B^{\prime \prime} \cap V_{i}$ are separated by $-q$ ).

Step 3. $A^{\prime \prime}$ and $B^{\prime \prime}$ can be separated in a neighbourhood of $\Gamma$.

It is possible to choose a semialgebraic neighbourhood $T$ of $\Gamma$ such that $X^{\prime \prime} \cap$ $\bar{T} \subseteq X^{\prime \prime} \cap V$. We want to prove that $A^{\prime \prime}$ and $B^{\prime \prime}$ can be separated in $T$ by applying Proposition 1.3 to the compact sets $\overline{A^{\prime \prime} \cap T}$ and $\overline{B^{\prime \prime} \cap T}$.

Since $\overline{A^{\prime \prime}} \cap \overline{B^{\prime \prime}} \subseteq($ Sing $Y) \cup X^{\prime \prime}$, also $\overline{A^{\prime \prime} \cap T} \cap \overline{B^{\prime \prime} \cap T} \subseteq(\operatorname{Sing} Y) \cup X^{\prime \prime}$.

As for $X^{\prime \prime}$, let $U^{\prime \prime}$ be a neighbourhood of $X^{\prime \prime}$ such that $U^{\prime \prime} \cap \bar{T} \subseteq V$. By Step 2, we have

$$
q\left(\overline{A^{\prime \prime} \cap T} \cap U^{\prime \prime}-X^{\prime \prime}\right)>0 \text { and } q\left(\overline{B^{\prime \prime} \cap T} \cap U^{\prime \prime}-X^{\prime \prime}\right)<0 .
$$

For each irreducible component $T_{j}$ of $\operatorname{Sing} Y$, by condition 2), there exists a regular function $p_{j}$ separating $A \cap U_{j}$ from $B \cap U_{j}$, i.e.

$$
p_{j}\left(A \cap U_{j}-Z\right)>0 \quad p_{j}\left(B \cap U_{j}-Z\right)<0 .
$$

Then

$$
p_{j} g^{\prime \prime}\left(A^{\prime \prime} \cap U_{j}-Z\right)>0 \quad p, g^{\prime \prime}\left(B^{\prime \prime} \cap U_{j}-Z\right)<0 .
$$

From (*) we get that $p_{j} g^{\prime \prime}$ separates $A^{\prime \prime} \cap U$, from $B^{\prime \prime} \cap U_{j}$. Recall that no irreducible component of $Y$ is odd w.r.t. $A^{\prime \prime}, B^{\prime \prime}$, so $\left(Y^{\prime \prime}\right)^{c} \subseteq X^{\prime \prime}$. So, if we apply Corollary 3.5 to $A^{\prime \prime} \cap T \cap U_{j}$ and $B^{\prime \prime} \cap T \cap U_{j}$, we get that, for each $j$, there exists a regular function $p^{\prime \prime}$ separating $A^{\prime \prime} \cap T \cap U_{j}$ from $B^{\prime \prime} \cap T \cap U_{j}$ and such that

$$
\begin{aligned}
& p_{j}^{\prime \prime}\left(\overline{A^{\prime \prime} \cap T} \cap U_{j}-\left((\operatorname{Sing} Y) \cup X^{\prime \prime}\right)\right)>0 \\
& p_{j}^{\prime \prime}\left(\overline{B^{\prime \prime} \cap T} \cap U_{j}-\left((\operatorname{Sing} Y) \cup X^{\prime \prime}\right)\right)<0 .
\end{aligned}
$$

This allows us to apply Proposition 1.3 to the compact sets $\overline{A^{\prime \prime} \cap T}$ and $\overline{B^{\prime \prime} \cap T}$ relatively to the algebraic set ( $\operatorname{Sing} Y) \cup X^{\prime \prime}$ : we get a function $\varphi$ which separates $A^{\prime \prime}$ from $B^{\prime \prime}$ in the neighbourhood $T$.

Step 4. $A$ and $B$ can be separated in a neighbourhood of $\Gamma$.

Coming back to $A$ and $B$, it follows from Step 3 that 


$$
\begin{aligned}
& \varphi g^{\prime \prime}\left(A \cap T-\left((\operatorname{Sing} Y) \cup X^{\prime \prime} \cup V\left(g^{\prime \prime}\right)\right)\right)>0 \\
& \varphi g^{\prime \prime}\left(B \cap T-\left((\operatorname{Sing} Y) \cup X^{\prime \prime} \cup V\left(g^{\prime \prime}\right)\right)\right)<0 .
\end{aligned}
$$

that is $A \cap T$ and $B \cap T$ can be generically separated. Because of condition 3), Corollary 3.5 implies that $A \cap T$ and $B \cap T$ can be separated by a regular function, we will denote $p_{T}$.

Let $g^{\prime}$ be a generator of the ideal $\mathscr{g}\left(Y^{c} \cup X^{\prime}\right)$ and consider the sets $A^{\prime}=A_{g^{\prime}}$ and $B^{\prime}=B_{g^{\prime}}$. Arguing as above, we can see that

$$
Z^{\prime} \cap\left(A^{\prime} \cup B^{\prime}\right)=Z \cap\left(A^{\prime} \cup B^{\prime}\right) .
$$

Step 5. $A^{\prime}$ and $B^{\prime}$ can be separated in a neighbourhood of $Y^{c}$.

Let $\Omega$ be a semialgebraic neighbourhood of $Y^{c}$ such that $\bar{\Omega} \cap X^{\prime} \subseteq T \cap X^{\prime}$. We want to prove that $A^{\prime}$ and $B^{\prime}$ can be separated in $\Omega$ by applying Proposition 1.3 to the compact sets $\overline{A^{\prime} \cap \Omega}$ and $\overline{B^{\prime} \cap \Omega}$.

Since $\overline{A^{\prime}} \cap \overline{B^{\prime}} \subseteq(\operatorname{Sing} Y) \cup X^{\prime}$, we have also $\overline{A^{\prime} \cap \Omega} \cap \overline{B^{\prime} \cap T} \subseteq(\operatorname{Sing} Y)$ $\cup X^{\prime}$.

As for $X^{\prime}$, let $U^{\prime}$ be a neighbourhood of $X^{\prime}$ such that $U^{\prime} \cap \bar{\Omega} \subseteq T$. By Step 4, $p_{T}$ separates $A \cap T$ from $B \cap T$; hence

$$
p_{T} g^{\prime}\left(\left(A^{\prime} \cap T\right)-Z\right)>0 \quad p_{T} g^{\prime}\left(\left(B^{\prime} \cap T\right)-Z\right)<0 .
$$

From (**), we get that $p_{T} g^{\prime}$ separates $A^{\prime} \cap T$ from $B^{\prime} \cap T$.

As before, we see that $\left(Y^{\prime}\right)^{c} \subseteq X^{\prime}$. So, if we apply Corollary 3.5 to $A^{\prime} \cap T$ and $B^{\prime} \cap T$, we get that there exists a regular function $p^{\prime}$ separating $A^{\prime} \cap T$ from $B^{\prime} \cap T$ and such that

$$
p^{\prime}\left(\overline{A^{\prime} \cap T}-\left(\operatorname{Sing} Y \cup X^{\prime}\right)\right)>0 \quad p^{\prime}\left(\overline{B^{\prime} \cap T}-\left(\operatorname{Sing} Y \cup X^{\prime}\right)\right)<0 .
$$

Now, since $U^{\prime} \cap \bar{\Omega} \subseteq T$, we have

$$
p^{\prime}\left(\overline{A^{\prime} \cap \Omega} \cap U^{\prime}-\left(\operatorname{Sing} Y \cup X^{\prime}\right)\right)>0 \quad p^{\prime}\left(\overline{B^{\prime} \cap \Omega} \cap U^{\prime}-\left(\operatorname{Sing} Y \cup X^{\prime}\right)\right)<0 \text {, }
$$

that is the hypothesis of Proposition 1.3 is fulfilled in the neighbourhood $U^{\prime}$ of $X^{\prime}$ with respect to the algebraic set ( $\operatorname{Sing} Y) \cup X^{\prime}$.

We have to prove that the hypothesis is satisfied also around each irreducible component $T$, of $\operatorname{Sing} Y$.

Arguing as in Step 3, from condition 2) we get that $p_{j} g^{\prime}$ separates $A^{\prime} \cap U_{j}$ from $B^{\prime} \cap U_{j}$. Since $\left(Y^{\prime}\right)^{c} \subseteq X^{\prime}$, if we apply Corollary 3.5 to $A^{\prime} \cap \Omega \cap U_{j}$ and $B^{\prime} \cap \Omega \cap U_{\text {, we }}$, wet that there exists a regular function $p^{\prime}$, separating $A^{\prime} \cap \Omega \cap$ $U_{j}$ from $B^{\prime} \cap \Omega \cap U_{j}$ and such that 
$p^{\prime}\left(\overline{A^{\prime} \cap \Omega} \cap U_{j}-\left(\operatorname{Sing} Y \cup X^{\prime}\right)\right)>0 \quad p^{\prime}\left(\overline{B^{\prime} \cap \Omega} \cap U_{j}-\left(\operatorname{Sing} Y \cup X^{\prime}\right)\right)<0$.

We can therefore apply Proposition 1.3 to the compact sets $\overline{A^{\prime} \cap \Omega}$ and $\overline{B^{\prime} \cap \Omega}$ relatively to the algebraic set $(\operatorname{Sing} Y) \cup X^{\prime}$ : we get a function $\phi$ which separates $A^{\prime}$ from $B^{\prime}$ in the neighbourhood $\Omega$.

Step 6. $A$ and $B$ can be separated in a neighbourhood of $Y^{c}$.

Coming back again to $A$ and $B$, from Step 5 it follows that

$$
\begin{gathered}
\psi g^{\prime}\left(\overline{A \cap \Omega}-\left(\operatorname{Sing} Y \cup X^{\prime} \cup V\left(g^{\prime}\right)\right)\right)>0 \\
\psi g^{\prime}\left(\overline{B \cap \Omega}-\left(\operatorname{Sing} Y \cup X^{\prime} \cup V\left(g^{\prime}\right)\right)\right)<0,
\end{gathered}
$$

that is $A \cap \Omega$ and $B \cap \Omega$ can be generically separated. Because of condition 3 ), Corollary 3.5 assures that $A \cap \Omega$ and $B \cap \Omega$ can be separated by a regular function, say $p_{\Omega}$.

Step 7. $A$ and $B$ can be separated.

We want to apply Proposition 1.3 to $\bar{A}$ and $\bar{B}$ relatively to $Y^{c} \cup Z$. In the neighbourhood $\Omega$ of $Y^{c}$, by Corollary 3.5 we may assume that

$$
p_{\Omega}\left(\bar{A} \cap \Omega-\left(Y^{c} \cup Z\right)\right)>0 \quad p_{\Omega}\left(\bar{B} \cap \Omega-\left(Y^{c} \cup Z\right)\right)<0 .
$$

As for $Z$, it is enough to consider its irreducible components $T_{j}$ not contained in $Y^{c}$ and therefore contained in Sing $Y$. Using condition 2) and again Corollary 3.5, we get that the hypothesis of Proposition 1.3 is verified also around $T_{j}$, and so we get a function $p$ such that

$$
p\left(\bar{A}-\left(Y^{c} \cup Z\right)\right)>0 \text { and } p\left(\bar{B}-\left(Y^{c} \cup Z\right)\right)<0 .
$$

Then, by condition 3 ),

$$
p(A-Z)>0 \text { and } p(B-Z)<0 .
$$

Remark 4.1. In the proof of Theorem 2.7, we actually separate $\bar{A}$ and $\bar{B}$ up to $W=Z \cup Y^{\mathrm{c}}$, which is "minimal" in the sense of Theorem 3.3 and Corollary 3.5. So if $F, G$ are closed semialgebraic sets such that $F=\overline{\bar{F}}, G=\overline{\bar{G}}$ and verifying conditions 1$), 2), 3)$, then there exists $p \in \mathscr{R}(M)$ such that

$$
p(F-W)>0 \quad p(G-W)<0 .
$$




\section{A separation criterion}

In this section we look for a criterion that makes it easier to decide whether $A$ and $B$ can be separated.

Consider, at first, the case in which the algebraic set $Y$ is a union of non-singular normal crossings components $Y_{1}, \ldots, Y_{k}$, each one of dimension 2 . Assume also that $Y_{\alpha} \cap Y_{\beta}$ is irreducible for any $\alpha \neq \beta$.

The test we are going to describe relates the separation of $A$ and $B$ with the separation or their two-dimensional "traces" on each irreducible component $Y_{\alpha}$ of $Y$, that is the sets

$$
\operatorname{tr}_{\alpha} A=\widehat{\stackrel{\circ}{\bar{A} \cap Y_{\alpha}}} \quad \operatorname{tr}_{\alpha} B=\widehat{\stackrel{\circ}{\bar{B} \cap Y_{\alpha}}}
$$

where the interior part is taken in $Y_{\alpha}$.

If $f \in \mathscr{J}\left(Y_{\alpha}\right)$ changes its sign across $Y_{\alpha}$, we have to consider also the traces of the sets $A_{f}$ and $B_{f}$.

Definition 5.1. Let $C, D$ be open semialgebraic subsets of $M$. We will say that the triple $\left(C, D, Y_{\alpha}\right)$ satisfies the property $(\mathrm{P})$ if the sets $\operatorname{tr}_{\alpha} C$ and $\operatorname{tr}_{\alpha} D$ are disjoint and can be separated in $Y_{\alpha}$. We will say that it satisfies the property $\left(\mathrm{P}^{\prime}\right)$ if $\left(C_{f}, D_{f}, Y_{\alpha}\right)$ verifies $(\mathrm{P})$, where $f$ is an element in $g\left(Y_{\alpha}\right)$ that changes its sign across $Y_{\alpha}$.

It is easy to verify that the property $\left(\mathrm{P}^{\prime}\right)$ does not depend on the choice of $f$ : suppose that both $f$ and $g$ change their sign across $Y_{\alpha}$; if $q$ separates $\operatorname{tr}_{\alpha} C_{f}$ from $\operatorname{tr}_{\alpha} D_{f}$, then $q f g$, reduced modulo $\mathscr{g}\left(Y_{\alpha}\right)^{2}$, generically separates $\operatorname{tr}_{\alpha} C_{g}$ from $\operatorname{tr}_{\alpha} D_{g}$, so (being in dimension 2) they can be separated.

We begin by proving the following

Lemma 5.2. The statements:

i) " $Y_{\alpha}$ is odd (resp. even)"

ii) " $\left(A, B, Y_{\alpha}\right)$ verifies $(\mathrm{P})$ (resp. $\left.\left(\mathrm{P}^{\prime}\right)\right)$ ”

cannot hold simultaneously.

Proof. Suppose, by contradiction, $Y_{\alpha}$ is odd and $\operatorname{tr}_{\alpha} A, \operatorname{tr}_{\alpha} B$ are disjoint and can be separated by a regular function $q$.

Then there exists an open semialgebraic subset $\Omega$ of $M$ such that $\operatorname{dim}\left(Y_{\alpha} \cap \Omega\right)$ 
$=2$ and $A \cap \Omega$ and $B \cap \Omega$ can be generically separated, say by $f \in \mathscr{R}(M)$, that is

$$
f(A \cap \Omega-X)>0 \text { and } f(B \cap \Omega-X)<0 .
$$

By the same argument already used in the proof of Theorem 3.3, we can assume $\operatorname{dim} X \leq 1$.

The functions $f$ and $q$ have the same sign on $U-Y_{\alpha}$, where $U \subseteq \Omega$ is a suitable semialgebraic neighbourhood of $\left(\operatorname{tr}_{\alpha} A \cup \operatorname{tr}_{\alpha} B\right) \cap \Omega-X$.

Define

$$
S=(\bar{A} \cup \bar{B}) \cap \bar{\Omega}-U
$$

it is a closed semialgebraic set and $\operatorname{dim}\left(S \cap Y_{\alpha}\right) \leq 1$.

Applying Theorem 3.1 to $f, q$ and $S$, we get a regular function $p=f+\varepsilon q$ which separates $A \cap \Omega-X$ from $B \cap \Omega-X$ and does not vanish on $Y_{\alpha}$. In fact:

- on $((A \cup B)-X) \cap \Omega \cap S, p$ and $f$ have the same sign,

- on $((A \cup B)-X) \cap \Omega-S$, which is contained in $U, f$ and $q$ have the same sign, so again $p$ and $f$ have the same sign.

Therefore $p$ separates $A \cap \Omega-X$ from $B \cap \Omega-X$.

Moreover $p \notin g\left(Y_{\alpha}\right)$; in fact, otherwise, $\varepsilon$ should identically vanish on $Y_{\alpha}$, which is impossible since $V(\varepsilon) \subseteq \overline{V(f) \cap S}^{Z}$ and $\operatorname{dim}\left(S \cap Y_{\alpha}\right) \leq 1$.

So $p$ does not change its sign across $Y_{\alpha}$ and $Y_{\alpha}$ is not odd. Contradiction.

To complete the proof, let $f \in \mathscr{J}\left(Y_{\alpha}\right)$ be a regular function that changes its sign across $Y_{\alpha}$. Then it is enough to remark that $Y_{\alpha}$ is even w.r.t. $A, B$ if and only if $Y_{\alpha}$ is odd w. r. t. $A_{f}, B_{f}$ (Lemma 2.5) and that $\left(A, B, Y_{\alpha}\right)$ verifies $\left(\mathrm{P}^{\prime}\right)$ if and only if $\left(A_{f}, B_{f}, Y_{\alpha}\right)$ verifies $(\mathrm{P})$. The first part of the proof yields the thesis.

Theorem 5.3. Let $A$ and $B$ be open disjoint semialgebraic sets. Assume that $Y=\overline{\partial A}^{Z} \cup \overline{\partial B}^{Z}$ is a union of non-singular irreducible components $Y_{1}, \ldots, Y_{k}$ of dimention 2, simultaneously normal crossings and such that $Y_{\alpha} \cap Y_{\beta}$ is irreducible for $\alpha \neq \beta$. Then $A$ and $B$ can be separated if and only if for each $\alpha \in\{1, \ldots, k\}(A, B$, $\left.Y_{\alpha}\right)$ verifies at least one between the property $(\mathrm{P})$ and the property $\left(\mathrm{P}^{\prime}\right)$.

Proof. $\quad(\Rightarrow)$ Assume that $A$ and $B$ can be separated and suppose, by contradiction, there exists $\alpha$ such that $\left(A, B, Y_{\alpha}\right)$ verifies neither $(\mathrm{P})$ nor $\left(\mathrm{P}^{\prime}\right)$.

We want to see that, since $\left(A, B, Y_{\alpha}\right)$ does not verify $(\mathrm{P})$, then $Y_{\alpha}$ is odd. This is clear if $\operatorname{tr}_{\alpha} A$ and $\operatorname{tr}_{\alpha} B$ are not disjoint. In the case they are disjoint, but not separated, let $g$ be a generator of $\mathscr{g}\left(Y_{\alpha}\right)^{2}$; for any regular function $p$ generically separating $A$ from $B$, we can write $p=g^{h} \cdot q$, with $q \notin g\left(Y_{\alpha}\right)^{2}$. Nevertheless 
$q \in \mathscr{J}\left(Y_{\alpha}\right)$, otherwise it would generically separate $\operatorname{tr}_{\alpha} A$ from $\operatorname{tr}_{\alpha} B$, which is impossible since in dimension 2 generic separation is equivalent to separation. The functions $p$ and $q$ have the same sign, so $p$ changes its sign across $Y_{\alpha}$ and hence $Y_{\alpha}$ is odd.

Arguing as before, we see that, since $\left(A, B, Y_{\alpha}\right)$ does not verify $\left(\mathrm{P}^{\prime}\right)$, then $Y_{\alpha}$ is even. Contradiction.

$(\Leftarrow)$ Assume that, for each $\alpha \in\{1, \ldots, k\},\left(A, B, Y_{\alpha}\right)$ verifies $(\mathrm{P})$ or $\left(\mathrm{P}^{\prime}\right)$. Then by Lemma 5.2 there are no 2-obstructions. Since $Y^{c} \cap(A \cup B)=\varnothing$ (see Remark 2.9), in order to apply Theorem 2.7 we have only to show that $A$ and $B$ can be separated in a neighbourhood $U$, of each irreducible component $T$, of Sing $Y$.

This can be done by modifying a little the proof of Theorem 2.7; let us give a sketch of it.

Let $Y_{\alpha}$ be an irreducible component of $Y$ containing $T_{j}$ and assume, for instance, that $\left(A, B, Y_{\alpha}\right)$ verifies (P). The curve $H={\overline{\partial \operatorname{tr}_{\alpha} A}}^{Z} \cup{\overline{\partial \operatorname{tr}_{\alpha} B}}^{Z}$ has non-singular, normal crossing, irreducible components, say $H_{1}, \ldots, H_{q}$ and for each $i=1, \ldots, q$ there exists by hypothesis an irreducible component $Y_{i}$ such that $Y_{i} \cap Y_{\alpha}=H_{i}$.

Denote by $H_{1}, \ldots, H_{s}$ the irreducible components of $H$ lying in $\overline{\overline{\operatorname{tr}_{\alpha} A} \cap \overline{\operatorname{tr}_{\alpha} B}} Z$. We can find an algebraic subset $X$ of $M$ such that $\left[Y_{1} \cup \ldots \cup Y_{s} \cup X\right]=0$ in $H_{2}\left(M, \mathbf{Z}_{2}\right)$ and such that $X$ is transversal to each irreducible component of $Y$ and of Sing $Y$.

Take a generator $g$ of $g\left(Y_{1} \cup \ldots \cup Y_{s} \cup X\right)$ and consider the sets $A_{g}$ and $B_{g}$ and their traces on $Y_{\alpha}$.

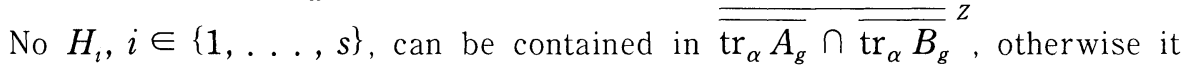
would be an obstruction to the separation of $\operatorname{tr}_{\alpha} A$ and $\operatorname{tr}_{\alpha} B$. So the set

$$
\left.\overline{\left(\operatorname{tr}_{\alpha} A_{g}\right.} \cap \overline{\operatorname{tr}_{\alpha} B_{g}}\right)-\left(X \cap Y_{\alpha}\right)
$$

is a finite set of points $\left\{Q_{1}, \ldots, Q_{h}\right\}$ with $Q_{i}=Y_{\alpha} \cap Y_{m(\imath)} \cap Y_{l(i)}$.

If we denote by $\Gamma_{i}$ the curve $Y_{m(\imath)} \cap Y_{l(\imath)}$ and if the neighbourhood $U_{j}$ is small enough, we have

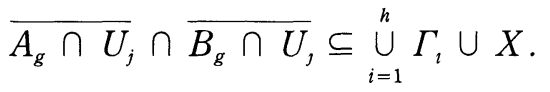

We want to apply Proposition 1.3 to the sets $\bar{A}_{g} \cap U$, and $\bar{B}_{g} \cap U_{j}$ and the algebraic set $\cup_{i=1}^{h} \Gamma_{\imath} \cup X$. Arguing as in Step 3 of the proof of Theorem 2.7 we get that $\overline{A_{g} \cap U}$, and $\overline{B_{g} \cap U}$, can be separated in a neighbourhood of $X$. 
In a small neighbourhood $V_{i}$ of $\Gamma_{\imath} \cap U_{j}$ take local equations $f_{m(i)}, f_{l(i)}$ for $Y_{m(i)}$ and $Y_{l(i)}$. If $U_{j}$ is sufficiently small, in $U_{j}$ the $\Gamma_{i}$ 's are pairwise disjoint, so the function $p_{\imath}=f_{m(i)}+f_{l(i)}\left(\right.$ or $\left.-p_{i}\right)$ verifies

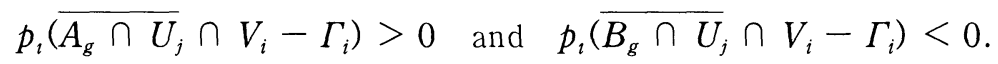

Since all the hypothesis of the Proposition 1.3 are fulfilled, we get that $A_{g} \cap U$, and $B_{g} \cap U_{j}$ can be separated by a regular function $p_{U_{j}}$. Then $p_{U_{j}} \cdot g$ generically separates $A \cap U_{j}$ from $B \cap U_{j}$ and therefore, as in Step 4, there exists a regular function separating them too. So also condition 2) of Theorem 2.7 is verified and therefore $A$ and $B$ can be separated.

Remark 5.4. It is easy to see that the "only if" part of Theorem 5.3 holds even if $Y$ is not normal crossings. Fig. 5 shows that the converse is not true in general.

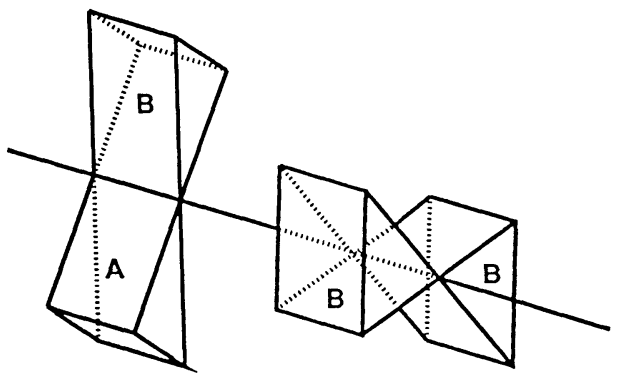

Fig. 5

Remark 5.5. If we now come back to the general situation (without the supplementary hypothesis on $Y$ considered before), we can make use of Theorem 5.3 as follows.

First of all consider a resolution of the singularities of $Y$, say $: \tilde{M} \rightarrow M$. Let $\tilde{Y}=\pi^{-1}(Y)$. By performing, if necessary, some further blowing-ups, we can suppose that $\tilde{Y}_{\alpha} \cap \tilde{Y}_{\beta}$ is irreducible, for any irreducible components $\tilde{Y}_{\alpha}, \tilde{Y}_{\beta}$ of $\tilde{Y}$, $\alpha \neq \beta$. We can also assume that $\tilde{Y}$ satisfies the hypothesis of Theorem 5.3 , because the 1-dimensional components of $\tilde{Y}$ can be "removed", as pointed out in Remark 3.4 .

Of course if $A$ and $B$ can be separated, then $\pi^{-1}(A)$ and $\pi^{-1}(B)$ can be separated too. Conversely we know (see Step 1 in the proof of Theorem 2.7) that if $\pi^{-1}(A)$ and $\pi^{-1}(B)$ can be separated, then $A$ and $B$ can be generically separated; if moreover $Y^{c} \cap(A \cup B) \subseteq Z$, they can be separated. 
Now we can test if $\pi^{-1}(A)$ and $\pi^{-1}(B)$ can be separated by means of Theorem 5.3, which therefore becomes a criterion of generic separation for $A$ and $B$. So Theorem 5.3 reduces the problem to a finite number of 2 -dimensional tests: the separation of the traces of $\pi^{-1}(A)$ and $\pi^{-1}(B)$. For that one can make use of the following result, analogous to Theorem 2.7:

Theorem 5.6. Let $M$ be a non-singular compact surface and $A$ and $B$ be open semialgebraic subsets of $M$. Then $A$ and $B$ can be separated if and only if:

a) No irreducible component of $Y=\bar{\partial}^{Z} \cup \overline{\partial B}^{Z}$ is both odd and even

b) $A$ and $B$ can be locally separated at any singular point of $Y$

In $[\mathrm{AcBgF}]$ one can find a proof of this result under a supplementary condition, which can be removed arguing as in Section 4; a direct and geometric proof that such condition is not necessary can be found in $[\mathrm{P}]$.

It is important to remark that, when applying Theorem 5.5 , one has to verify only condition a) of the theorem, because it is clear that in a normal crossings situation condition a) implies condition b).

\section{REFERENCES}

[AcBg] F. Acquistapace, F. Broglia, More about signatures and approximation, Geometriae Dedicata, 50 (1994), 107-116.

$[\mathrm{AcBgF}] \quad$ F. Acquistapace, F. Broglia, E. Fortuna, When is a distribution of signs locally completable? , Canad. J. Math., 46 (1994), 449-473.

[AnBrRz] C. Andradas, L. Bröcker, J. M. Ruiz, Constructible sets in real geometry, (1996), Erg. Math. 33, Springer-Verlag.

[BoCRy] J. Bocknak, M. Coste, M. F. Roy, Géométrie algébrique réelle, (1987), Springer-Verlag, Berlin-Heidelberg-New York.

[Br1] L. Bröcker, On the separation of basic semialgebraic sets by polynomials, Manuscripta Math., 60 (1988), 497-508.

[Br2] L. Bröcker, On basic semialgebraic sets, Exp. Math., 9 (1991), 289-334.

[F] E. Fortuna, Distribution de signes, Mathematika, 38 (1991), 50-62.

[P] L. Pernazza, Decidability of the separation problem in dim 2, to appear.

[Rz] J. M. Ruiz, A note on a separation problem, Arch. Math., 43 (1984), $422-426$. 
Dipartimento di Matematica

Università di Pisa

Via F. Buonarroti 2

56127 Pisa, Italy

E-mail:acquistf@dm.unipi.it

broglia@dm.unipi.it

fortuna@dm.unipi.it 